\title{
VISCO-ACOUSTIC MODELING IN THE FREQUENCY DOMAIN USING A MIXED-GRID FINITE-DIFFERENCE METHOD AND ATTENUATION-DISPERSION MODEL
}

\author{
Sheryl K. Avendaño ${ }^{1,2}$, Juan Carlos Muñoz-Cuartas ${ }^{1,2}$, Miguel A. Ospina² and Hebert Montegranario²
}

\begin{abstract}
Seismic modeling is an important step in the process used for imaging Earth sub-surface. Current applications require accurate models associated with solutions of the equation of wave propagation in realistic medium. In this work, we propose a modeling for 2D wave propagation in a visco-acoustic medium with variable velocity and density, handled in the frequency domain under conditions that describe dissipation depending on the quality factor $Q$. We use mixed-grid finite-difference method and optimize it for the case of the visco-acoustic medium with the aim to minimize numerical dispersion. We present solutions for test cases in homogeneous media and compare the analytic solutions. Further, we compare the solution using conventional grid (5-point scheme) and our mixed-grid implementation (9-point scheme), finding a better response with the mixed-grid 9-point scheme. We also studied the characteristics of the numerical solution, wave fields for P-waves are discussed for different velocity profiles, damping functions and $Q$ values finding that the method performs very well with potential in applications that require full knowledge of the wave field such as Full Waveform Inversion or Reverse Time Migration.
\end{abstract}

Keywords: seismic attenuation, wave propagation modeling, visco-acoustic medium, quality factor.

RESUMO. A modelagem sísmica é um passo importante no processo da construção de imagens da sub-superfície da Terra. Aplicações atuais exigem modelos de exatidão associados a soluções da equação de propagação de ondas em meio realista. Neste trabalho, nós propomos uma modelagem para propagação de ondas 2D em um meio visco-acústico com velocidade e densidade variáveis, manipuladas no domínio da frequência sob condições que descrevem a dissipação dependendo do fator de qualidade $Q$. Utilizamos o método de diferenças finitas em redes mistas e otimizamos para o caso do meio visco-acústico com o objetivo de minimizar a dispersão numérica. Apresentamos soluções para casos de teste em meios homogêneos e comparamos com as soluções analíticas. Além disso, comparamos a solução usando uma rede convencional (5-pontos) e nossa implementação de redes mistas (9-pontos), encontrando uma melhor resposta com o esquema de 9-pontos da rede mista. Também estudamos as características da solução numérica, campos de onda para ondas P são discutidos para diferentes perfis de velocidade, funções de amortecimento e valores de $Q$, descobrindo que o método funciona muito bem com potencial em aplicações que exigem conhecimento completo do campo de onda, como inversão de forma de onda completa ou Migração de Tempo Inverso.

Palavras-chave: atenuação sísimica, modelagem de propagação de onda, meio visco-acústico, fator de qualidade.

\footnotetext{
${ }^{1}$ Universidad de Antioquia, Group of Computational Physics and Astrophysics, Instituto de Física, Medellin, Calle 67 No. 53-108, Colombia - E-mails: sheryl.avendano@udea.edu.co, juan.munozc@udea.edu.co

2Universidad de Antioquia, Group of Tomography and Seismics, Instituto de Matemáticas, Medellin, Calle 67 No. 53-108, Colombia -E-mails: maospina5@gmail.com, hebert.montegranario@udea.edu.co
} 


\section{INTRODUCTION}

Realistic modeling of the propagation of waves in the continuous media is one of the most complex problems one faces in different scenarios of applied physics. Although this problem is complex, it is often a crucial step for the solution of many different situations, from the exploration of the inner structure of solids and medical imaging to seismic exploration. In the particular case of seismic exploration, modeling the wave propagation in the sub-surface is complex. In general, the physical conditions are non-ideal, therefore when one requires realistic, but still practical solutions to the problem, several simplifying approximations have to be made. For example, one usually ignores the effects of attenuation in wave propagation. However, Earth is not totally elastic neither totally viscous and a combination of both extreme behaviors should fit the properties of wave propagation.

The simplest approximation to wave propagation is the acoustic and isotropic case. However, when this approach is not enough to reproduce the observables, in order to get precise information about the physical properties of the sub-surface, it becomes necessary to consider a better model of the propagation of waves considering more realistic physical conditions such as viscosity, elasticity and anisotropy.

Viscosity and attenuation of mechanical waves are key physical phenomena and we shall focus our attention in this subject. The visco-acoustic media can be defined as a medium without cross propagation but exhibiting attenuation in the amplitude of the longitudinal wave. Considering viscosity in wave propagation introduces two phenomena not present in the acoustic case. First, dissipation produced by energy absorption such that the amplitude of the wave is reduced especially at high frequency. Second, dispersion by the change in the physical properties of the medium, where the wave velocity may depend on the frequency (Wang, 2009).

To describe the attenuation of energy of the seismic wavefront, Carcione et al. (2002) proposed a model based in linear solid material rheology and memory variables. They show in their work how the use of memory variables describes the propagation of waves in the medium and accounts for attenuation of the energy of the propagating waves at large distances from the source. Later, Dutta et al. (2013) used the same modeling approach, however, using only one relaxation mechanism, they show how the method works to compensate for attenuation in least-squares reverse time migration (LSRTM).

Wang et al. (2003) used a visco-acoustic wave equation to compensate for the energy decrease of wave propagation in a realistic media using an extrapolator based in the propagator of the wave equation in the forward and backward direction. They show that using a $Q$-based attenuation model it is possible to fully describe the propagation of waves in the visco-acoustic media. Yang et al. (2014) present a detailed comparison of different visco-acoustic wave equations and studied their dissipation and dispersion properties, providing a rough idea about how the most commonly used visco-acoustic wave equations perform.

In particular, the realistic modeling of wave propagation is an important step in the process used for imaging Earth sub-surface. Currently, one of the most powerful techniques of seismic tomography that uses solutions of the equation of wave motion is the so-called Full Waveform Inversion (FWI) (Fichtner, 2011). FWI consists on obtaining the model parameters of the sub-surface through an inversion process that relates the comparison between models and observed data. To understand and emulate the complex wave phenomena in a realistic heterogeneous medium it is necessary to perform a seismic modeling where the result is a wavefield obtained through the numerical solution of the wave propagation.

This previous background shows that for different scenarios in seismic exploration it is still important to study the problem of wave propagation in visco-acoustic media. However, the numerical solution of the differential equation of wave propagation requires high accuracy, this translates in to a problem where large matrix operations are involved and this implies the demand of large computational resources. This consideration introduces expensive constraints from the technical point of view. The goal now then is to look for methods that provide high quality solutions that offer the opportunity to explode the computational power available to date.

We aim to provide a model for the propagation of waves in a general visco-acoustic media in the frequency domain optimized for applications in FWI (Full Waveform Inversion), considering the effects of attenuation. It is one more solution to visco-acoustic wave equation but this time in frequency domain. There have been several proposals to include the effects of attenuation in the equations of wave motion (Yang et al., 2014). One possibility is to work in the frequency domain using a term known as complex bulk modulus, which is a method easy to implement and to relate to the quality factor $Q$ (Carcione et al., 2002). Other works use Laplace transform for the model (Kamei \& Pratt, 2013) and FWI (Shin \& Ho Cha, 2008). 
This work focuses on the propagation of mechanical waves in a medium with explicit attenuation modeled through the damping functions and quality factor $Q$. Frequency domain is adopted in this work since it allows the study of effects of the modeling (attenuation, dispersion) on individual wave frequencies. We pay especial attention to the quality of the solution and provide results of the implementation of optimal mixed-grid scheme looking for the minimization of the numerical dispersion in the implementation. Instead modeling explicitly the elastic and viscous excitation modes (Carcione et al., 2002), we model the dissipative effect of the medium explicitly through damping functions that attenuate the wave amplitude according to the quality factor of the media for different frequencies.

\section{METHODOLOGY}

\section{Background and mathematical and physical formulation}

Wave propagation rests on the basis of mechanics of the continuum medium. In order to formulate the problem we need to state a set of basic equations that describe the motion of the media and perturbations propagating inside it

$$
\begin{gathered}
\left(\frac{\partial}{\partial t}+\mathbf{v}(\mathbf{x}, t) \cdot \nabla\right) \rho(\mathbf{x}, t)+\rho(\mathbf{x}, t) \nabla \cdot \mathbf{v}(\mathbf{x}, t)=0 . \\
\rho(\mathbf{x}, t)\left(\frac{\partial}{\partial t}+\mathbf{v}(\mathbf{x}, t) \cdot \nabla\right) \mathbf{v}(\mathbf{x}, t)=\nabla \cdot \boldsymbol{\sigma}(\mathbf{x}, t)+\mathbf{f}(\mathbf{x}, t), \quad \int_{V} \varepsilon_{i j k} \sigma_{j k}(\mathbf{x}, t) d V=0 . \\
\sigma_{i j}=\varepsilon_{k l}(\mathbf{x}, t) \circledast \dot{C}_{i j k l}(\mathbf{x}, t) .
\end{gathered}
$$

Equation (1) is the continuity equation, that is an statement of the conservation of mass, Equation (2) is an establishment of linear and angular momentum conservation, and Equation (3) is a governing equation. In these equations $\rho$ is the density, $\mathbf{v}$ represents the particle velocity, $\boldsymbol{\sigma}$ is the stress tensor, $\mathbf{f}$ represents the body forces, $\varepsilon$ is a Levi-Civita tensor, $\boldsymbol{C}$ is the elastic tensor, $\boldsymbol{\varepsilon}$ is the strain tensor and $\circledast$ is a convolution operator (Mase \& Mase, 1999). Now, if we assume that the medium is visco-acoustic, irrotational and compressible but the flow is approximately constant and wave propagation is only longitudinal and there are no shear waves, we can reduce the governing equation, Equation (3) to

$$
\sigma_{i j}(\mathbf{x}, t)=-p(\mathbf{x}, t) \delta_{i j}=\nabla \cdot \mathbf{u}(\mathbf{x}, t) \circledast M(\mathbf{x}, t) \delta_{i j},
$$

where $p(\mathbf{x}, t)$ is a pressure wave, $\mathbf{u}(\mathbf{x}, t)$ is the particle displacement, $\delta_{i j}$ is a Kronecker delta and $M(\mathbf{x}, t)$ is the time derivative of the elastic tensor often named complex bulk modulus (Fichtner, 2011). $M(\mathbf{x}, t)$ accounts for the elastic and dissipative properties of the media. Using Equation (4) one can obtain Hooke's law and combining Eqs. (2) and (3) one can get Newton's law.

$$
\begin{gathered}
p(\mathbf{x}, t)=-\nabla \cdot \mathbf{u}(\mathbf{x}, t) \circledast M(\mathbf{x}, t) . \\
\left(\frac{\partial}{\partial t}+\gamma(\mathbf{x})\right) \mathbf{v}=\frac{1}{\rho(\mathbf{x})}(\nabla \cdot \boldsymbol{\sigma}+\mathbf{f}),
\end{gathered}
$$

where $\gamma(\mathbf{x})$ is called the rate deformation function, such that it represents the temporal variation of the stress tensor and is related to the viscosity of the medium. It is an important term in the relation of energy stored and energy lost per cycle (Wang, 2009). Applying a Fourier transform to Equations (5) and (6) we have

$$
\begin{gathered}
-i \omega P(\omega, \mathbf{x})=M(\omega, \mathbf{x}) \nabla \cdot \mathbf{V}(\omega, \mathbf{x}) . \\
-i \omega \mathbf{V}(\omega, \mathbf{x})=-\frac{b(\mathbf{x})}{\xi(\omega, \mathbf{x})} \nabla P(\omega, \mathbf{x})+\frac{b(\mathbf{x})}{\xi(\omega, \mathbf{x})} \mathbf{F}(\omega, \mathbf{x}),
\end{gathered}
$$

where $\xi(\omega, \mathbf{x})=1-i \frac{\gamma(\mathbf{x})}{\omega}$ and $b(\mathbf{x})=\frac{1}{\rho(\mathbf{x})}$. The propagation of waves in a visco-acoustic media presents both dissipation and dispersion. These effects are modeled using the damping functions. The complex bulk modulus must be related to $\xi(\omega, \mathbf{x})$ subject to the constraint that when no dissipation $M(\omega, \mathbf{x}) \rightarrow K(\omega, \mathbf{x})$, where $K(\omega, \mathbf{x})$ is an acoustic bulk modulus. So we define

$$
M(\omega, \mathbf{x})=K(\mathbf{x}) \alpha(\omega, \mathbf{x})=K(\mathbf{x}) \frac{1}{\xi(\omega, \mathbf{x})},
$$


where $\alpha(\omega, \mathbf{x})$ is complex. To establish a relation between $\alpha$ and $\xi$ we combine the Equations (7) and (8) in the homogeneous case, with $\rho=$ cte, $c=$ cte and $\gamma=$ cte. If $\alpha=\xi$ this reduces to the acoustic wave equation in frequency domain, but since $\alpha=\frac{1}{\xi}$ then the equation is recast as

$$
\left(\tilde{k}^{2}+\nabla^{2}\right) P=\nabla \cdot \mathbf{F} .
$$

Equation (10) is the Helmholtz equation with complex coefficients, where $\tilde{k}$ is called complex wavenumber. The real part of $\tilde{k}$ is $\frac{\omega}{c}$, the conventional wavenumber, and the imaginary part $\frac{\gamma}{c}$ is the pseudo-wavenumber. This concept is applied in attenuation-dispersion models related to the quality factor $Q$.

\section{Equation of propagation in a visco-acoustic medium}

Then, the equation for wave propagation in the visco-acoustic media can be obtained combining Equations (7), (8) and (9) as

$$
\frac{\omega^{2} P(\omega, \mathbf{x})}{K(\mathbf{x})}+\frac{1}{\xi(\omega, \mathbf{x})} \nabla \cdot\left(\frac{b(\mathbf{x})}{\xi(\omega, \mathbf{x})} \nabla P(\omega, \mathbf{x})\right)=\frac{1}{\xi(\omega, \mathbf{x})} \nabla \cdot\left(\frac{b(\mathbf{x})}{\xi(\omega, \mathbf{x})} \mathbf{F}(\omega, \mathbf{x})\right) .
$$

In this situation we have the freedom to choose the way we model the attenuation, and one can then, for instance, use analytic models for this function (e.g. Wang, 2009). Wang (2009) present different models where they define the wavenumber as $k(\omega)=$ $\frac{\omega}{c(\omega)}=\frac{\omega}{v(\omega)}-i \kappa(\omega)$, where $c(\omega)$ is the complex velocity, $v(\omega)$ the phase velocity and $\kappa(\omega)$ the attenuation wavenumber, rewriting in terms of $\xi$, it can be shown like

$$
\xi(\omega, \mathbf{x}) \cong \frac{c}{v(\omega)}-i \frac{\kappa(\omega) c}{\omega} \approx 1-i \frac{\kappa(\omega) c}{\omega},
$$

where $v(\omega) \sim c$. According to this, in this work we present results using the modified Kolsky's model for attenuation-dispersion (Kolsky, 2003)

$$
\kappa(\omega, \mathbf{x})=\frac{|\omega|}{2 Q(\mathbf{x}) c(\mathbf{x})} ; \quad \frac{1}{v(\omega, \mathbf{x})}=\frac{1}{c(\mathbf{x})}\left(1-\frac{1}{\pi Q(\mathbf{x})} \ln \left|\frac{\omega}{\omega_{h}}\right|\right),
$$

where $\omega_{h}$ is the highest possible seismic frequency, about $1000 \pi \mathrm{Hz}$. Substituting Equations 12 in the last relation for $\xi(\omega, \mathbf{x})$, and considering that for values of $Q$ and $\omega$ in the range of interest $(Q \in[30,650], \omega \in[2 \pi, 1000 \pi])$ the term $\frac{1}{\pi Q} \ln \left|\frac{\omega}{\omega_{h}}\right|$ vanishes (Wang, 2009), then the damping function can be simplified to

$$
\xi(\omega, \mathbf{x}) \approx 1-i \frac{1}{2 Q(\mathbf{x})},
$$

which leads to a very simple model of attenuation that is only dependent on the $Q$ factor that can enter as a new parameter in the model.

\section{Numerical solution technique}

In general, obtaining a solution of Equation (11) is not possible for arbitrary medium properties and boundaries. A natural approach is then to look for numerical solutions using e.g. finite-differences. Assuming a 2D problem with coordinates $(x, z)$ the problem can be re-written as

$$
\begin{gathered}
-i \omega P(\omega, \mathbf{x})=K(\mathbf{x}) \frac{1}{\xi(\omega, \mathbf{x})}\left(\frac{\partial V_{x}(\omega, \mathbf{x})}{\partial x}+\frac{\partial V_{z}(\omega, \mathbf{x})}{\partial z}\right), \\
-i \omega V_{x}(\omega, \mathbf{x})=-\frac{b(\mathbf{x})}{\xi(\omega, \mathbf{x})} \frac{\partial P(\omega, \mathbf{x})}{\partial x}+S_{x}(\omega, \mathbf{x}) \\
-i \omega V_{z}(\omega, \mathbf{x})=-\frac{b(\mathbf{x})}{\xi(\omega, \mathbf{x})} \frac{\partial P(\omega, \mathbf{x})}{\partial z}+S_{z}(\omega, \mathbf{x})
\end{gathered}
$$


where $S_{x}(\omega, \mathbf{x})=\nabla \cdot\left(\frac{b(\mathbf{x})}{\xi(\omega, \mathbf{x})} F_{x}(\omega, \mathbf{x})\right)$ and $S_{z}(\omega, \mathbf{x})=\nabla \cdot\left(\frac{b(\mathbf{x})}{\xi(\omega, \mathbf{x})} F_{z}(\omega, \mathbf{x})\right)$. Although the finite-difference method is widely used for solving differential equations, it has very well known problems of numerical dispersion that are related to the discretization of the media. Jo (1996) propose a scheme of two overlapping grids to solve the PDE aimed to avoid these numerical dispersion problems. Although Jo (1996) presents its method for the solution of acoustic problems, we go a step further and implement the ideas for the solution of our problem of the visco-acoustic wave equation. In Jo (1996), one of the grids is a horizontally oriented cartesian grid, while the second one is a rotated cartesian grid (see Fig. 1). In this kind of setup, the solution is obtained evaluating the fields in a total of 9 grid points, thus reducing the numerical dispersion and trying to cover all directions of propagation.
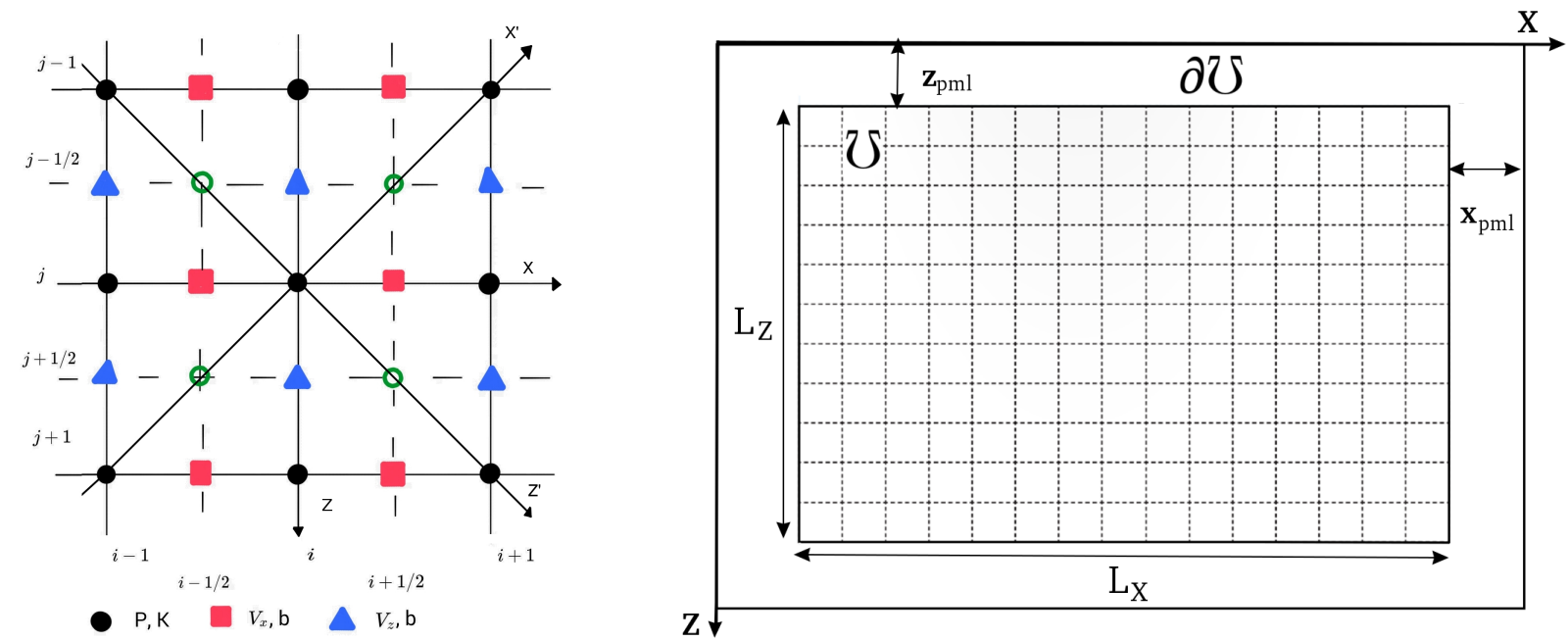

Figure 1 - (Left) Illustration of the mixed-grid used in the numerical solution. The black points represent the field pressure in the conventional grid, the triangular and square symbols represent the field particle velocity in the intercalated grid, the diagonals represent the axes for the rotated grid. The partial derivatives are computed for points in conventional grid and rotated grid, in total 9 points. (Right) Workspace $\mho$ and boundary workspace $\partial \mho$ for the numerical solution.

\section{Mixed-Grid and Lumped Mass strategy}

Then, using centered finite-differences for partial derivatives, in the conventional grid, the derivatives of the function $\mathbf{V}(\mathbf{x}, \omega)$ and $P(\mathbf{x}, \omega)$ are expressed as

$$
\begin{aligned}
& \left.\frac{\partial V_{x}}{\partial x}\right|_{(i, j)} \approx \frac{1}{\Delta}\left(V_{x(i+1 / 2, j)}-V_{x(i-1 / 2, j)}\right),\left.\quad \frac{\partial V_{z}}{\partial z}\right|_{(i, j)} \approx \frac{1}{\Delta}\left(V_{z(i, j+1 / 2)}-V_{x(i, j-1 / 2)}\right) . \\
& \left.\frac{\partial P}{\partial x}\right|_{(i \pm 1 / 2, j)} \approx \frac{1}{\Delta}\left( \pm P_{(i \pm 1, j)} \mp P_{(i, j)}\right),\left.\quad \frac{\partial P}{\partial z}\right|_{(i, j \pm 1 / 2)} \approx \frac{1}{\Delta}\left( \pm P_{(i, j \pm 1)} \mp P_{(i, j)}\right),
\end{aligned}
$$

where $\Delta$ is the grid spacing. Now, for the rotated grid, using a rotation of $45^{\circ}$, we obtain the transformation rule for the derivatives in that system such that centered finite-differences of partial derivatives, in the rotated grid of $\mathbf{V}(\mathbf{x}, \omega)$ and $P(\mathbf{x}, \omega)$ are

$$
\begin{gathered}
\left.\frac{\partial V_{x}}{\partial x^{\prime}}\right|_{(i, j)} \approx \frac{1}{\sqrt{2} \Delta}\left(V_{x(i+1 / 2, j-1 / 2)}-V_{x(i-1 / 2, j+1 / 2)}\right), \\
\left.\frac{\partial V_{z}}{\partial z^{\prime}}\right|_{(i, j)} \approx \frac{1}{\sqrt{2} \Delta}\left(V_{z(i+1 / 2, j+1 / 2)}-V_{x(i-1 / 2, j-1 / 2)}\right) . \\
\left.\frac{\partial P}{\partial x^{\prime}}\right|_{(i \pm 1 / 2, j \neq 1 / 2)} \approx \frac{1}{\sqrt{2} \Delta}\left( \pm P_{(i \pm 1, j \mp 1)} \mp P_{(i, j)}\right),
\end{gathered}
$$




$$
\left.\frac{\partial P}{\partial z^{\prime}}\right|_{(i \pm 1 / 2, j \pm 1 / 2)} \approx \frac{1}{\sqrt{2} \Delta}\left( \pm P_{(i \pm 1, j \pm 1)} \mp P_{(i, j)}\right) .
$$

To improve the accuracy of the mixed-grid stencil the pressure acceleration term $\frac{\omega^{2} P(\omega, \mathbf{x})}{K(\mathbf{x})}$ may not be taken as a term implying only the collocation point (the so-called lumped approximation). The implementation of the mixed-grid aims to reduce the numerical dispersion taking 9 points to find the values of the derivatives, if the free term $\frac{\omega^{2} P(\omega, \mathbf{x})}{K(\mathbf{x})}$ takes only the value at point $(i, j), P_{i, j}$, the contribution of the rotated grid will not be considered and the homogeneity of the solution will be lost to some degree (Operto \& Virieux, 2006) and the effort of using a rotated grid may be wasted.

In order to solve this issue we may approximate this term by using a weighted average over the mixed operator stencil nodes, in a similar way as it is done in finite-element modeling (see e.g. Marfurt, 1984 or Strikwerda, 2007). Then in this situation the lumped mass term shall take the form

$$
\left.\frac{\omega^{2} P(\omega, \mathbf{x})}{K(\mathbf{x})}\right|_{(i, j)}=\frac{\omega^{2}}{K_{(i, j)}}\left(m_{2} \Sigma_{1(i, j)} P+\frac{m_{4}}{4} \Sigma_{2(i, j)} P\right)
$$

with $\Sigma_{1(i, j)} P=\frac{1}{4}\left(P_{i+1, j}+P_{i-1, j}+P_{i, j+1}+P_{i, j-1}\right), \Sigma_{2(i, j)} P=\frac{1}{4}\left(P_{i+1, j-1}+P_{i-1, j-1}+P_{i-1, j+1}+P_{i+1, j+1}\right)$ and $m_{4}=1-m 2+4 m_{3}$, where $m_{2}$ and $m_{3}$ are coefficients regulating the relation between the contribution of the terms by the conventional and rotated grid in the lumped mass term. The other terms in the conventional grid and in the rotated grid are given by

$$
\left.\frac{1}{\xi(\omega, \mathbf{x})} \nabla \cdot\left(\frac{b(\mathbf{x})}{\xi(\omega, \mathbf{x})} \nabla P(\omega, \mathbf{x})\right)\right|_{(i, j)}=\Gamma_{(i, j)} P,\left.\quad \frac{1}{\xi(\omega, \mathbf{x})} \nabla^{\prime} \cdot\left(\frac{b(\mathbf{x})}{\xi(\omega, \mathbf{x})} \nabla^{\prime} P(\omega, \mathbf{x})\right)\right|_{(i, j)}=\Theta_{(i, j)} P,
$$

where the notation $\nabla^{\prime}$ stands for derivatives in the rotated system. Then, the final version of the discretization of Equation (12) is

$$
m_{1} \Gamma_{(i, j)} P+\left(1-m_{1}\right) \Theta_{(i, j)} P+\frac{\omega^{2}}{K_{(i, j)}}\left(m_{2} \Sigma_{1(i, j)} P+\frac{m_{4}}{4} \Sigma_{2(i, j)} P\right)=\left.S(\omega, \mathbf{x})\right|_{(i, j)},
$$

where $m_{1}$ is a coefficient regulating the relation between the contribution of the terms by the conventional and rotated grid but in the remaining divergence terms in Equation (11).

As it is well known, one of the advantages of the solution of the problem of wave propagation in frequency domain is that at the end, the problem can be formulated as a linear problem for each single frequency $\omega$. In our case, that linear problem shall have the form $\mathbf{A p}=\mathbf{s}$, where the matrix $\mathbf{A}$ is given by

$$
\begin{aligned}
& A_{(m, n)}=D_{(m)} \delta_{m, n}+D 1_{(m)} \delta_{m, n+N_{z}}+D 2_{(m)} \delta_{m, n-N_{z}}+D 3_{(m)} \delta_{m, n+1} \\
& +D 4_{(m)} \delta_{m, n-1}+D 5_{(m)} \delta_{m, n+N_{z}-1}+D 6_{(m)} \delta_{m, n-N_{z}+1}+D 7_{(m)} \delta_{m, n+N_{z}+1} \\
& +D 8_{(m)} \delta_{m, n-N_{z}-1},
\end{aligned}
$$

with the coefficients $D l_{(i, j)}$, for $l=0,1,2, \ldots 8, m=i N_{z}+j, i=0,1,2 \ldots N_{x}-1$ and $j=0,1,2 \ldots N_{z}-1$ given by

$$
\begin{aligned}
& D l_{(i, j)}=\frac{\omega^{2}}{K_{i, j}} A_{l}\left(m_{2}, m_{3}, m_{4}\right)+B_{l}\left(\eta_{x(i, j)}^{ \pm}, \eta_{z(i, j)}^{ \pm}\right) m_{1} \\
& +C_{l}\left(\eta_{x 1(i, j)}^{ \pm}, \eta_{x 2(i, j)}^{ \pm}, \eta_{z 1(i, j)}^{ \pm}, \eta_{z 2(i, j)}^{ \pm}\right)\left(1-m_{1}\right),
\end{aligned}
$$

where the functions $A_{l}, B_{l}$ and $C_{l}$ can be seen explicitly in Avendaño (2017) and terms $\eta$ have the form

$$
\begin{array}{cl}
\eta_{x(i, j)}^{ \pm}=\frac{1}{\xi_{(i, j)}} \frac{1}{\Delta^{2}} \frac{b_{(i \pm 1 / 2, j)}}{\xi_{(i \pm 1 / 2, j)}} ; & \eta_{z(i, j)}^{ \pm}=\frac{1}{\xi_{(i, j)}} \frac{1}{\Delta^{2}} \frac{b_{(i, j \pm 1 / 2)}}{\xi_{(i, j \pm 1 / 2)}} ; \\
\eta_{x 1(i, j)}^{ \pm}=\frac{1}{\xi_{(i, j)}} \frac{1}{4 \Delta^{2}} \frac{b_{(i \pm 1 / 2, j \mp 1 / 2)}}{\xi_{(i \pm 1 / 2, j)}} ; & \eta_{z 1(i, j)}^{\mp}=\frac{1}{\xi_{(i, j)}} \frac{1}{4 \Delta^{2}} \frac{b_{(i \pm 1 / 2, j \neq 1 / 2)}}{\xi_{(i, j \neq 1 / 2)}} ; \\
\eta_{x 2(i, j)}^{ \pm}=\frac{1}{\xi_{(i, j)}} \frac{1}{4 \Delta^{2}} \frac{b_{(i \pm 1 / 2, j \pm 1 / 2)}}{\xi_{(i \pm 1 / 2, j)}} ; & \eta_{z 2(i, j)}^{ \pm}=\frac{1}{\xi_{(i, j)}} \frac{1}{4 \Delta^{2}} \frac{b_{(i \pm 1 / 2, j \pm 1 / 2)}}{\xi_{(i, j \pm 1 / 2)}} .
\end{array}
$$


This matrix is sparse, indefinite, complex and square. The vector $s$ in this linear problem is the source vector discretized around the coordinates $\left(x_{s}, z_{s}\right)$. For cases when the velocity, density and attenuation are not constant we propose that the source should have the form

$$
S_{(i, j)}=\frac{b_{(i, j)}}{\xi_{(i, j)}^{2}} R_{0} \frac{2}{\sqrt{\pi}} \frac{f^{2}}{f_{s}^{3}} e^{-\frac{f^{2}}{f_{s}^{2}}} \delta_{i, i_{s}} \delta_{j, j_{s}},
$$

where $R_{0}$ is the maximum amplitude of the Ricker wavelet, $f_{s}$ the source frequency, $\left(i_{s}, j_{s}\right)$ is a point in the grid where the source is placed and $\boldsymbol{\delta}_{i, i_{s}} \boldsymbol{\delta}_{j, j_{s}}$ can be approximated as

$$
\delta_{i, i_{s}} \delta_{j, j_{s}} \approx \frac{1}{\pi \sigma_{x} \sigma_{z}} e^{-\left(\frac{x_{i}-x_{s}}{\sigma_{x}}\right)^{2}} e^{-\left(\frac{z_{j}-z_{s}}{\sigma_{z}}\right)^{2}} .
$$

This definition comes from the Dirac delta function as the limit of the sequence of zero-centered normal distributions $\delta_{\sigma}(x)=$ $\frac{1}{\sqrt{\pi} \sigma} \exp ^{-\left(\frac{x}{\sigma}\right)^{2}}$ as $\sigma \rightarrow 0$. The factor $\sigma$ must have a relationship with the grid spacing $\Delta$, i.e. $\sigma_{x}=\sigma_{z}=s \Delta$, where $s \in[0.75,1.0]$ (see Avendaño, 2017). Finding $\mathbf{p}$ as the solution to the problem stated in equation $\mathbf{A p}=\mathbf{s}$ is equivalent to solve the system of Equations (11).

\section{Dispersion Analysis}

As it is clear from the use of finite-differences, the numerical solution requires a discretization to represent the physical domain that in our case is implemented in a regular mesh which has both a finite physical extent and finite number of mesh points. However, the number of points per unit wavelength that the approximate field encounters is not the same in every direction, and this artificial artifact due to the discreteness adds to the numerical dispersion of the solution. Clearly, this is not a problem that can be ignored. Since the solution is not exact, there is already an error on the approach, if we add this harmful effect to the solution, we will have, specially at long wavelengths, an inappropriate estimate of the solution due to the undersampling where long wavelengths are not sampled at high enough spatial frequencies to provide a trustable solution at those wavelengths (Deraemaeker \& Bouillard, 1999; Ospina, 2016).

In this part we will make use of the know behavior of the problem of wave propagation in a homogeneous media (Eq. 10). In this case for constant velocity, density and attenuation in a media without source we have that the wave equation (Eq. 10) may be written as

$$
\left(\nabla^{2}+\tilde{k}^{2}\right) P\left(x_{i}, z_{j}\right) \approx m_{1} \Gamma_{(i, j)} P+\left(1-m_{1}\right) \Theta_{(i, j)} P+\Phi\left(m_{2}, m_{3}\right)_{(i, j)} P=0,
$$

where $m_{1}, m_{2}, m_{3}$ are parameters that can be found by minimizing the dispersion of the solution. To do so, remember that in this case $\rho$ and $c$ have a constant value, then the problem has an analytic solution given by $P(r)=P_{0} e^{-i(\tilde{\mathbf{k}} \cdot \mathbf{r})}$. Where $\tilde{\mathbf{k}}$ is a complex wave vector and $\mathbf{r}$ is the position vector. Now, If we introduce the solution into the numerical scheme in Equation (33), we see that the numeric complex wavenumber can be written as the ratio of two functions $A$ and $B$ depending on the number of points per wavelength, $G_{r}$, and wave propagation angle $\boldsymbol{\theta}$ (see Jo, 1996; Ospina, 2016) as

$$
\tilde{k}^{2}=\frac{1}{\Delta^{2}} \frac{A\left(\tau_{r}, \tau_{i}, \theta\right)}{B\left(\tau_{r}, \tau_{i}, \theta\right)}
$$

where $\tau_{r}=\frac{1}{G_{r}}$ is the number of wavelengths per grid point. The same relation holds for the pseudo grid number for pseudo wavelength $G_{i}$ and $\tau_{i}=\frac{1}{G_{i}}$. With this notation, the relation between the complex wavenumber, complex wavelength and complex grid number is $\tilde{k}=\frac{\omega}{c}+i \frac{\gamma}{c} ; \quad \tilde{\lambda}=\Delta G_{r}+i \Delta G_{i} ; \quad \tilde{k}=\frac{2 \pi}{\Delta G_{r}}+i \frac{2 \pi}{\Delta G_{i}}$. 
Taking the square root of Equation (34), dividing by the theoretical estimate of $\tilde{k}, \tilde{k}^{T}$ and separating in to real and imaginary parts one gets

$$
N\left(\tau_{r}, \tau_{i}, \theta\right)=\frac{k_{r}^{N}}{k_{r}^{T}}=\frac{1}{2 \pi \tau_{r}} \mathfrak{R}\left(\sqrt{\frac{A}{B}}\right) ; \quad M\left(\tau_{r}, \tau_{i}, \theta\right)=\frac{k_{i}^{N}}{k_{i}^{T}}=\frac{1}{2 \pi \tau_{i}} \mathfrak{I}\left(\sqrt{\frac{A}{B}}\right) .
$$

Then, our objective function is

$$
\chi\left(m_{1}, m_{2}, m_{3}\right)=\iiint\left[\left(1-N\left(\tau_{r}, \tau_{i}, \theta\right)\right)^{2}+\left(1-M\left(\tau_{r}, \tau_{i}, \theta\right)\right)^{2}\right] d \theta d \tau_{r} d \tau_{i},
$$

which we try to minimize in the range of values $\theta \in[0, \pi / 2]$ and $\tau_{r}, \tau_{i} \in[0.001,0.15]$ for the parameters $m_{1}, m_{2}$ and $m_{3}$ (Chen, 2004). The values of the parameters that minimize the dispersion are then $m_{1}=0.6667, m_{2}=0.6556, m_{3}=0.0889$. It is important to mention that when there is no attenuation, we get the same result presented in other works $m_{1}=0.5461, m_{2}=0.6248, m_{3}=$ $0.09381(\mathrm{~J} 0,1996)$. Also using the values $m_{1}=1.0, m_{2}=1.0, m_{3}=0.0$ we can recover the usual 5 -point scheme.

To study the difference between the numerical dispersion produced by the scheme of 9-point and the scheme of 5-point, we plot in Figures 2 and 3 the relation between the theoretical and numeric wavenumbers for the real and imaginary parts, $k_{r}^{N} / k_{r}^{T}$ and $k_{i}^{N} / k_{i}^{T}$. We use the optimum values found in the minimization, and plot the behavior for different propagation angles, $\theta=0, \frac{\pi}{12}, \frac{\pi}{6}, \frac{\pi}{4}$ in the Equation (35). We calculate $M\left(\tau_{r}, \tau_{i}, \theta\right)$ for $\tau_{r}=0.142857$, and $\tau_{i} \in[0.001,0.15]$, and calculate $N\left(\tau_{r}, \tau_{i}, \theta\right)$ for $\tau_{i}=0.020$ and $\tau_{r} \in[0.001,0.15]$.

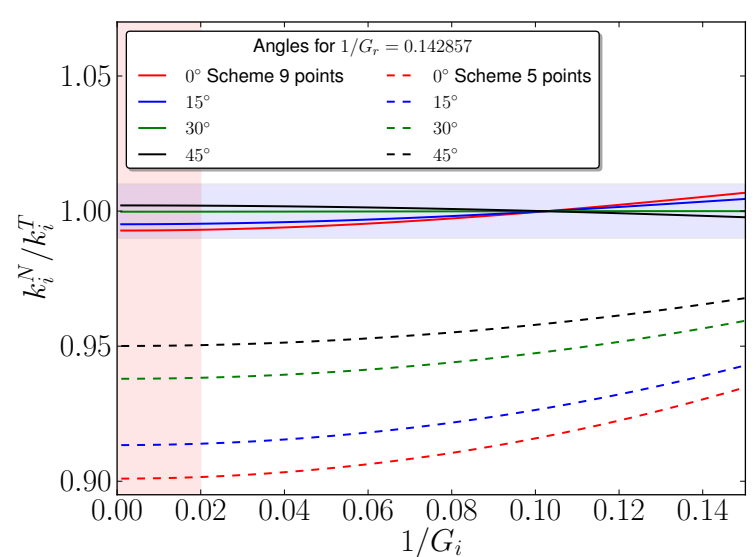

Figure 2 - Ratio between the imaginary part of theoretical and numerical wavenumber for $1 / G_{r}=0.142857$.

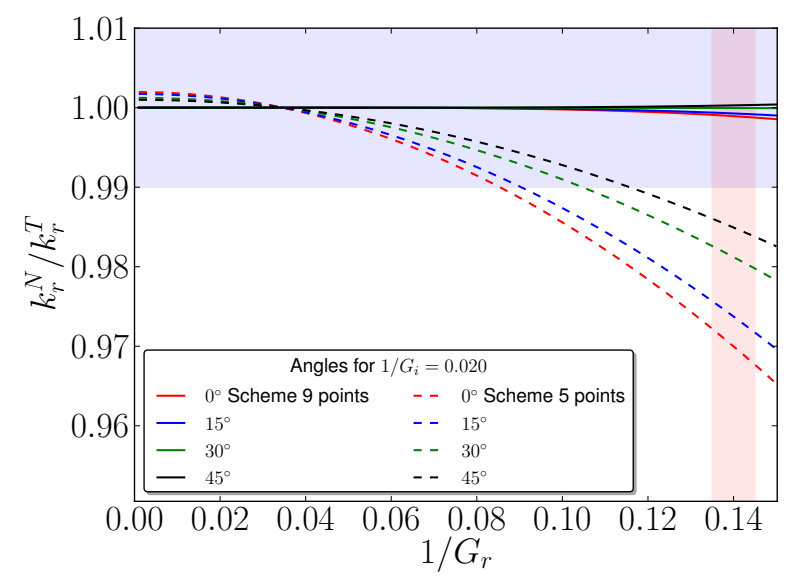

Figure 3 - Ratio between the real part of theoretical and numerical wavenumber for $1 / G_{i}=0.020$

To understand this figure, remember that the closer these ratios are to 1, the lower the numerical dispersion is and better the quality of the solution. The solid lines show the ratio computed for the scheme of 9-point while dashed lines show the ratio estimated for the 5-point scheme. The different lines for each scheme (different color lines) show the result for different propagation angles $\theta$.

Now, looking at the attenuation model discussed in Equation (13) one can show that for the value of the parameters used in this work and the physical conditions we explore, it will always be the case that $\lambda_{i}>\lambda_{r}$, then we always choose the approach to make $\Delta$ as a function of the wavelength and not of pseudo wavelength.

If $\tau_{r}=1 / 7$ (see Fig. 2), for any value of $\tau_{i}$, the dispersion is smaller for the 9-point scheme as the blue/horizontal highlighted region shows. Of course, if we choose the same $\Delta$ the expected values for $\tau_{i}$ are in the range $[0,0.02]$ when the dispersion is smaller as the red/vertical highlighted region shows. If $\tau_{i}=0.02$ (see Fig.3), for any value of $\tau_{r}$ the dispersion is smaller for the 9-point scheme as the blue/horizontal highlighted region shows, especially in the region $\tau_{r} \in[0.135,0.145]$ (red/vertical highlighted region). Now, according to the above, we define the cell size $\Delta=\frac{\lambda}{G_{r}}=\frac{c_{\min }}{f G_{r}}$. Where $G_{r}=7, c_{\min }$ is the lower wave velocity and $f$ is the frequency. 


\section{Perfectly-matched layer (PML) absorbing boundary conditions}

An important ingredient of the numerical solution are the boundary conditions. Since numerical solutions are constrained to a finite computational area (2D problem), border effects may introduce artificial boundaries or reflectors that are not real physical objects in the field. In order to account for these effects, the propagation of waves leaving the computational domain (the workspace) have to be considered with special care. Let us name our workspace as the region $\mho$ such that $\mho=\left\{(x, z) \in \mathfrak{R}^{2}: x \in\left[x_{p m l}, L_{x}+x_{p m l}\right] \wedge z \in\left[z_{p m l}, L_{z}+z_{p m l}\right]\right\}$. $\mho$ represents the region of the space where we are interested in to study the propagation of waves. And we extend the workspace with $\partial \mho=$ $\left\{(x, z) \in \mathfrak{R}^{2}: x \in\left[0, L_{x}+2 x_{p m l}\right] \wedge z \in\left[0, L_{z}+2 z_{p m l} \wedge(x, z) \notin \mho\right]\right\}$ and use Perfectly-Matched-Absorbing layer (PML) boundary conditions (Berenger, 1994) (see Fig. 1) to solve for the propagation of waves in this extended region. This boundary conditions in frequency domain uses two damping functions $\xi_{x}$ and $\xi_{z}$ which are related to $\xi$.

In the expanded region, the damping functions have the form Operto \& Virieux (2006)

$$
\begin{gathered}
\xi_{x}(\omega, \mathbf{x})=\left\{\begin{array}{cl}
1+i\left[\frac{\left.m_{0} \gamma_{x p m}\right]}{\omega}\right] & \text { If } x<x_{p m l} \quad \text { or } x>x_{p m l}+L_{x}, \\
\xi(\omega, \mathbf{x}) & \text { If } x_{p m l}<x<L_{x}+x_{p m l}
\end{array},\right. \\
\gamma_{x p m l}(\omega, \mathbf{x})=\left\{\begin{array}{cc}
\cos \left(\frac{x}{x_{p m l}} \frac{\pi}{2}\right)+\gamma\left(\omega, x_{p m l}, z\right) & \text { If } x<x_{p m l} \\
1-\cos \left(\frac{x-L_{x}-x_{p m l}}{x_{p m l}} \frac{\pi}{2}\right)+\gamma\left(\omega, L_{x}, z\right) & \text { If } x>x_{p m l}+L_{x}
\end{array},\right.
\end{gathered}
$$

where $m_{0}$ is a parameter that changes with frequency and takes a value that makes the amplitude of the wave at the boundary of the domain to fall below a given threshold (Avendaño, 2017). The PML for $z$ is defined in a similar way.

\section{RESULTS}

Once defined the optimal parameters for the discretization, we can model the propagation a P-wave in media with different velocity profiles and verify the performance of the numerical approach we propose. As a first test we model a Ricker pulse propagating in a medium with constant velocity, density and attenuation. The goal of this test is to compare with the solution of the Helmholtz equation with source that for this special case can be obtained analytically. Then we did several tests with different velocity profiles, the density in these cases, was calculated following the relation (Mavko et al., 2009)

$$
\rho(\mathbf{x})=\left\{\begin{array}{cc}
\sum_{i=1}^{5} a_{i} c(\mathbf{x})^{i} \mathrm{Kg} / \mathrm{m}^{3} & \text { If } c(\mathbf{x})>1480 \mathrm{~m} / \mathrm{s} \\
1050 \mathrm{Kg} / \mathrm{m}^{3} & \text { If } c(\mathbf{x})<1480 \mathrm{~m} / \mathrm{s}
\end{array}\right.
$$

where the constant parameters are $a_{1}=1.1 \times 10^{-16}, a_{2}=-4.3 \times 10^{-12}, a_{3}=6.7 \times 10^{-8}, a_{4}=-4.7 \times 10^{-4}$ and $a_{5}=1.66$. Besides this, the values for the quality factor associated with the different values of the velocity in the multiple layer media are modeled according to Lavergne (1986)

\section{Constant density, velocity, and attenuation}

In frequency domain, the Helmholtz equation with sources (Eq. 10) has an analytic solution given by Chen (2004)

$$
P(\omega, \mathbf{x}, \gamma)=i \pi H_{0}^{(2)}\left(\frac{\omega-i \gamma}{c} r\right) S(\omega, \gamma)
$$

where $S(\omega, \gamma)$ is a source, $r$ accounts for the distance between the source and any point with coordinates $(x, z)$ and is given by $r^{2}=\left(x-x_{s}\right)^{2}+\left(z-z_{s}\right)^{2}$. With $x_{s}$ and $z_{s}$ the position of source. 
In order to compare the results of this analytic solution with results obtained with the 9-point and 5-point schemes, we compute the value of the $P$-wave amplitude in an area of $2 \mathrm{~km} \times 2 \mathrm{~km}$ by placing a line of receivers at $100 \mathrm{~m}$ depth and a second line of receivers and $1100 \mathrm{~m}$ depth, finding the waveform response in those points. For this experiment the constant velocity of the media is $2100 \mathrm{~m} / \mathrm{s}$, the Ricker frequency is $30 \mathrm{~Hz}$, the constant quality factor is $Q=50$ using the damping function shown in Equation (13), the cell size $\Omega$ is $\lambda / G_{r}$, where $G_{r}=7$. The position of the source is $x_{0}=1 \mathrm{~km}$ and $z_{0}=1 \mathrm{~km}$.

In Figures 4 and 5 we show the normalized amplitude of the P-wave (real part) as a function of the offset for shots in the receivers at $1100 \mathrm{~m}$ depth and receivers at $100 \mathrm{~m}$ depth, respectively. They also show the difference between P-wave found by the analytic solution and 9-point scheme and 5-point scheme, at three different frequencies. Amplitudes are normalized dividing by its maximum value. In these figures the solid line is the analytic solution, the dashed line is the solution for the 5 -point scheme and the dotted line is the solution for 9-point scheme. It is worth to note that for all frequencies the solution obtained by the 9-point scheme is the closest to the analytic solution.

One can also see the strong disagreement of the solution obtained with the 5-point scheme compared to the analytic one, this can be seen especially at $\mathrm{P}$-waves away from the source. This is due mainly to numerical dispersion and grid point number, for 5 -point scheme (see Fig. 1) for $\tau_{r}=0.142857$ the relation between $k_{r}^{N} / k_{r}^{T}$ is about 0.95 and $k_{i}^{N} / k_{i}^{T}$ is about 0.98 , when the optimal value is close to 1.0. The dispersion for P-waves away from the source is more noticeable because the effect of pollution propagates with distance from the source, the numerical dispersion makes the P-wave to be out of phase.

In order to avoid such dispersion in the solution for the 5-point scheme, we would have to use a larger amount of points (Jo, 1996) and that would means a larger computational cost. In that sense we can conclude that the scheme of 9-point provides an appropriate solution at an acceptable computational cost.

\section{Media with three layers velocity profile}

The next test is performed with a different velocity profile and is performed in order to study the behavior of the solution in media with interfaces. In this experiment the P-wave field is computed in a media configured as the superposition of three parallel layers and all solutions are shown for the 9-point scheme.

In Figure 6 we show the real, imaginary parts and modulus of the P-wave field for frequency $5 \mathrm{~Hz}$. At that low frequency regime, the reflectors are not well defined due to the fact that the wavelength is too big compared with the features and the scale of the discretization, and one can not define the exact position of the reflectors or anomalies at scales smaller than the wavelength of the perturbation, however the oscillation is uniform.

In Figures 7 and 8, we show the same for frequencies of $25 \mathrm{~Hz}$ and $50 \mathrm{~Hz}$ respectively. Note that increasing the frequency the reflectors become more evident, but given that the gradient of the velocity is smooth there is continuity in the propagation of waves, as expected if we had relatively continuous profiles.

\section{Media with variable velocity, density and attenuation}

For this final test we decided to use a complex velocity profile. As it can be seen in Figure 9, in this case we have several wedge and important discontinuities with velocity contrast ranging from 1.0 to 3.0. For this setup the source is close to the surface at $15 \mathrm{~m}$ depth and again only the solutions with the 9-point scheme are shown.

In Figures 10, 11 and 12, we show the real and imaginary parts and modulus of the P-wave field for frequencies of 5, 25 and 50 $\mathrm{Hz}$. The result is less uniform than in the previous figures, revealing a natural response to the anisotropy of the velocity field. One can see how the features of the velocity field can distort the wavefront, even at large distances. Numerical dispersion have been minimized, so most of what can be seen is real response of the wave propagating inside the complex media.

\section{DISCUSSION AND CONCLUSIONS}

In this work we have studied the propagation of waves in a visco-acoustic medium through explicit modeling of the attenuation making use of damping functions that allow for dispersion that depends on the quality factor $Q$. We have implemented a finite-difference 

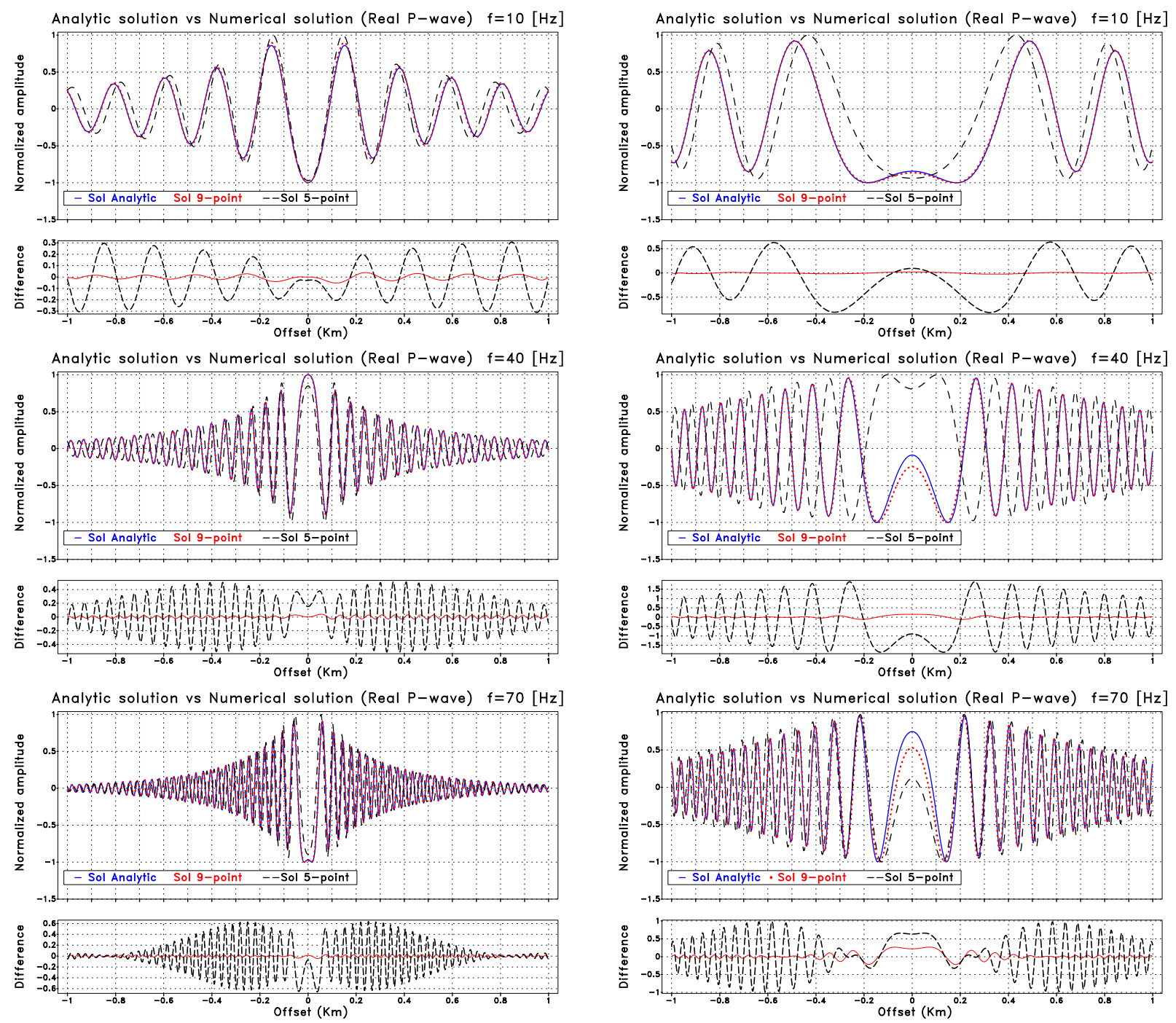

Figure 4 - Shots (real part) propagating in medium with constant velocity, density and attenuation for $f=10,40,70 \mathrm{~Hz}$ and receivers in $z=1100 \mathrm{~m}$ depth (close to the source in $z=1000 \mathrm{~m}$ ). The bottom panels show the difference of the 9-point (red) and 5-point (black) schemes against the exact solution.

Figure $\mathbf{5}$ - Shots (real part) propagating in medium with constant velocity, density and attenuation for $f=10,40,70 \mathrm{~Hz}$ and receivers in $z=100 \mathrm{~m}$ depth (away from the source in $z=1000 \mathrm{~m}$ ). The bottom panels show the difference of the 9-point (red) and 5-point (black) schemes against the exact solution.

scheme to solve the problem in frequency domain. Special care have been taken on the harmful numerical dispersion issues of the modeling, for which we have used a mixed-grid technique and optimal setup of the intercalated grids to minimize numeric dispersion.

We have shown the ability of the optimization scheme to minimize the numerical dispersion for the visco-acoustic case, and show that indeed the mixed-grid scheme (9-point) with the optimization scheme provides solutions that are very much close to the real solution than the solutions obtained for the classic 5-point scheme. Comparison of the numeric scheme with the analytic solution obtained for the case of wave propagation in a homogeneous medium has shown the advantages of the optimized mixed-grid scheme. As it was shown in Figures 1 and 5, the optimized 9-point mixed-grid scheme provides solutions that are in general a few percent away from the analytic solution, while the standard 5-point scheme deviates notoriously from the analytic solution even for this simplistic case. 

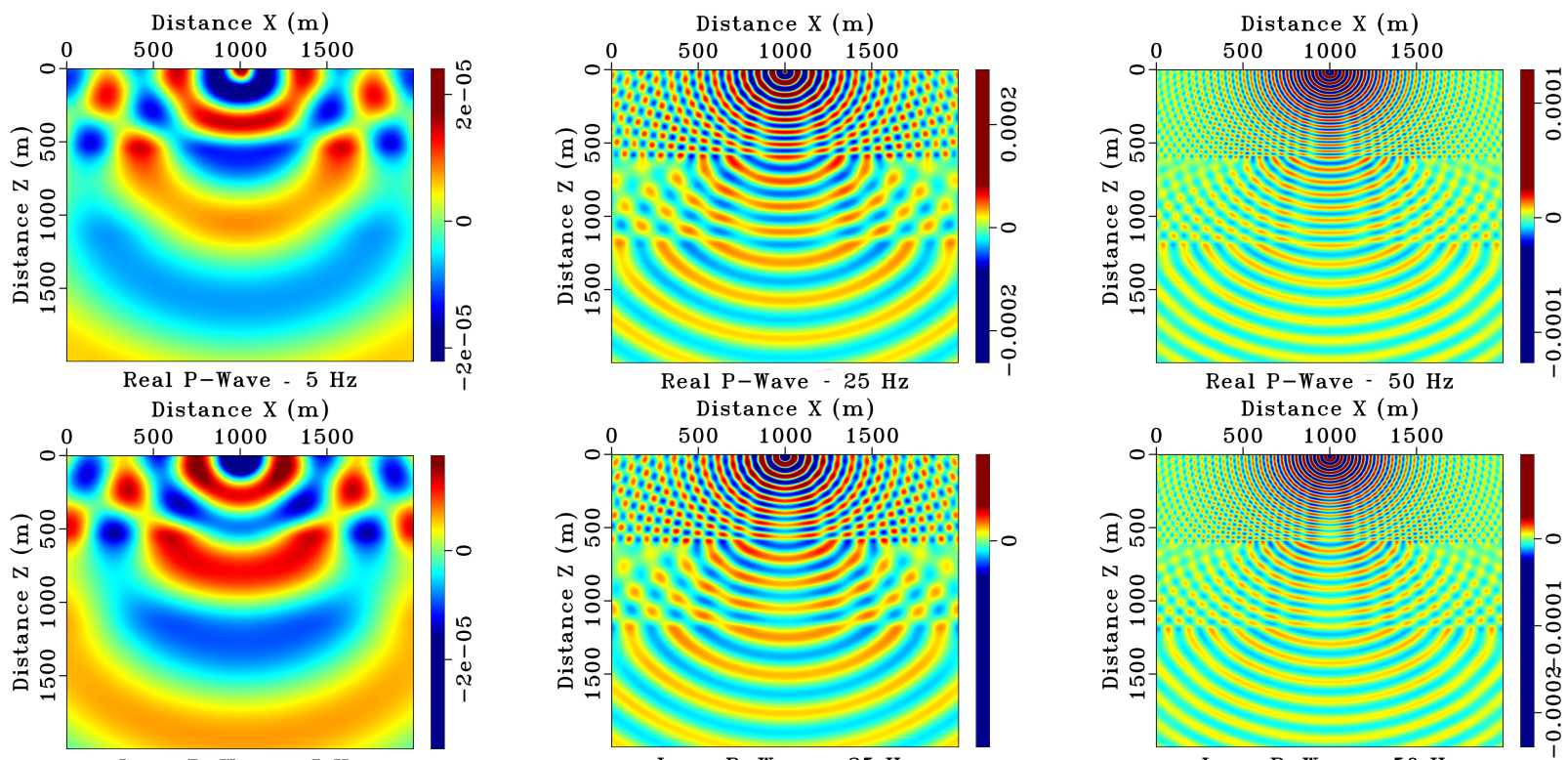

Imag P-Wave - $25 \mathrm{~Hz}$

Distance $X(\mathrm{~m})$

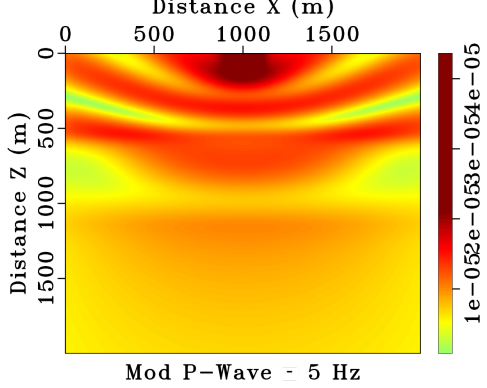

Distance X $(\mathrm{m})$

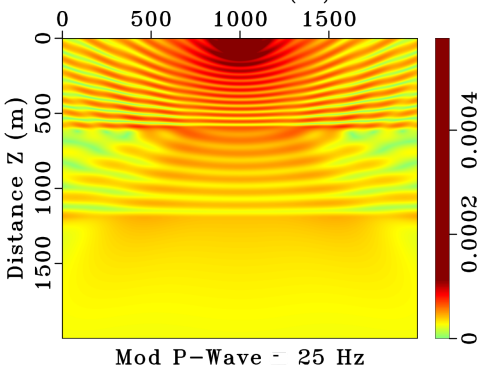

ag $\mathrm{P}-$ Wave - 50

Distance $X(\mathrm{~m})$

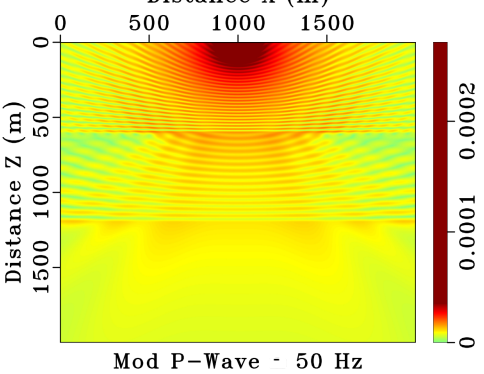

Figure 7 - Real, imaginary part and module at P-wave for $25 \mathrm{~Hz}$ with velocity field with three

Figure 8 - Real, imaginary part and module at P-wave for $50 \mathrm{~Hz}$ with velocity field with three parallel layers $\left(v_{1}=2100\right.$ in $[0,0.6] \mathrm{km}, v_{2}=$ $4300 \mathrm{~m} / \mathrm{s}$ in $(0.6,1.2] \mathrm{km}$ and $v_{3}=6500 \mathrm{~m} / \mathrm{s}$ in $(1.2,2.0] \mathrm{km})$ The source is close to the surface at $15 \mathrm{~m}$. $(1.2,2.0] \mathrm{km})$ The source is close to the surface at $15 \mathrm{~m}$.

We have also shown that this modeling works quite well in models of high velocity/density contrast, commonly found in nature. We have shown that the explicit modeling of attenuation allows to model easily the superposition of complex velocity fields and to resolve properly the behavior of waves in such scenarios. Although in this work we have focused in the results of the application of Kolski's model, we have verified the behavior of the method for other well known attenuation-dispersion models (Cole-Cole and general models; Wang, 2009), and in particular noticed the clear difference between the attenuation of the amplitude of the wavefront due to the distribution of energy in the expanding wavefront and the extra attenuation introduced by the damping functions.

This kind of behavior has a notorious importance for methods like FWI. In the case of seismic exploration, sub-surface information can be obtained through the use of Full Waveform Inversion. The quality of the information acquired through the inversion is of course dependent on the ability of the model to approach the physical properties of the medium. Visco-acoustic modeling represents a compromise between simplicity and realism that offers good results in the FWI technique, so, this kind of modeling has very promising impact in such procedures. In particular, given the iterative nature of $\mathrm{FWI}$, the use of optimized mixed-grid technique ensures the quality of the solution at reasonable computational cost. Another potential use is that an appropriated modeling of the attenuation may lead to better compensation for correct processing in e.g. RTM migration. 


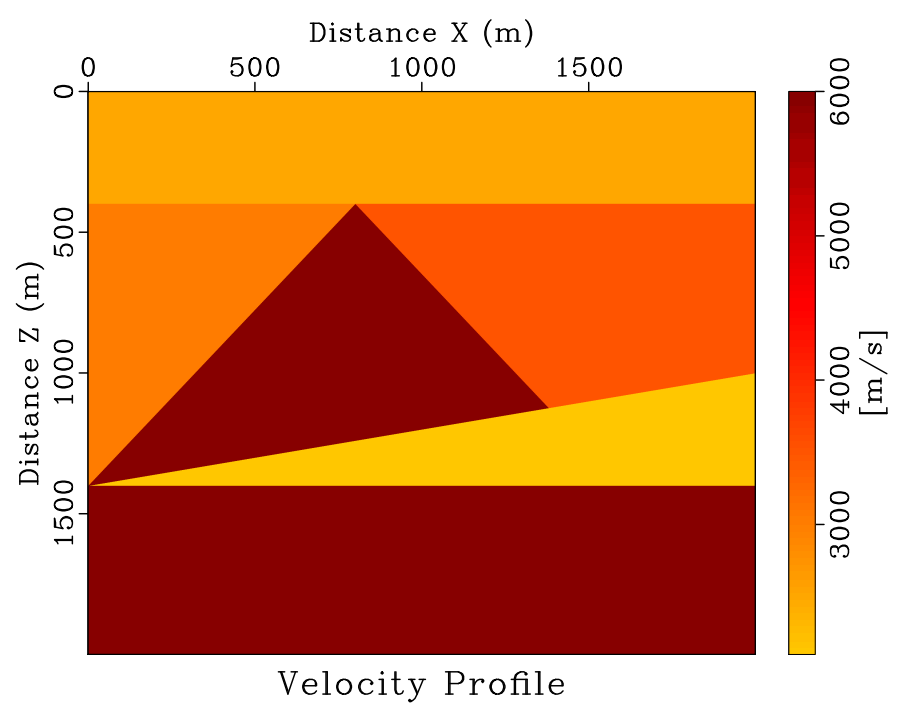

Figure $\mathbf{9}$ - Geometric configuration of the velocity profile.
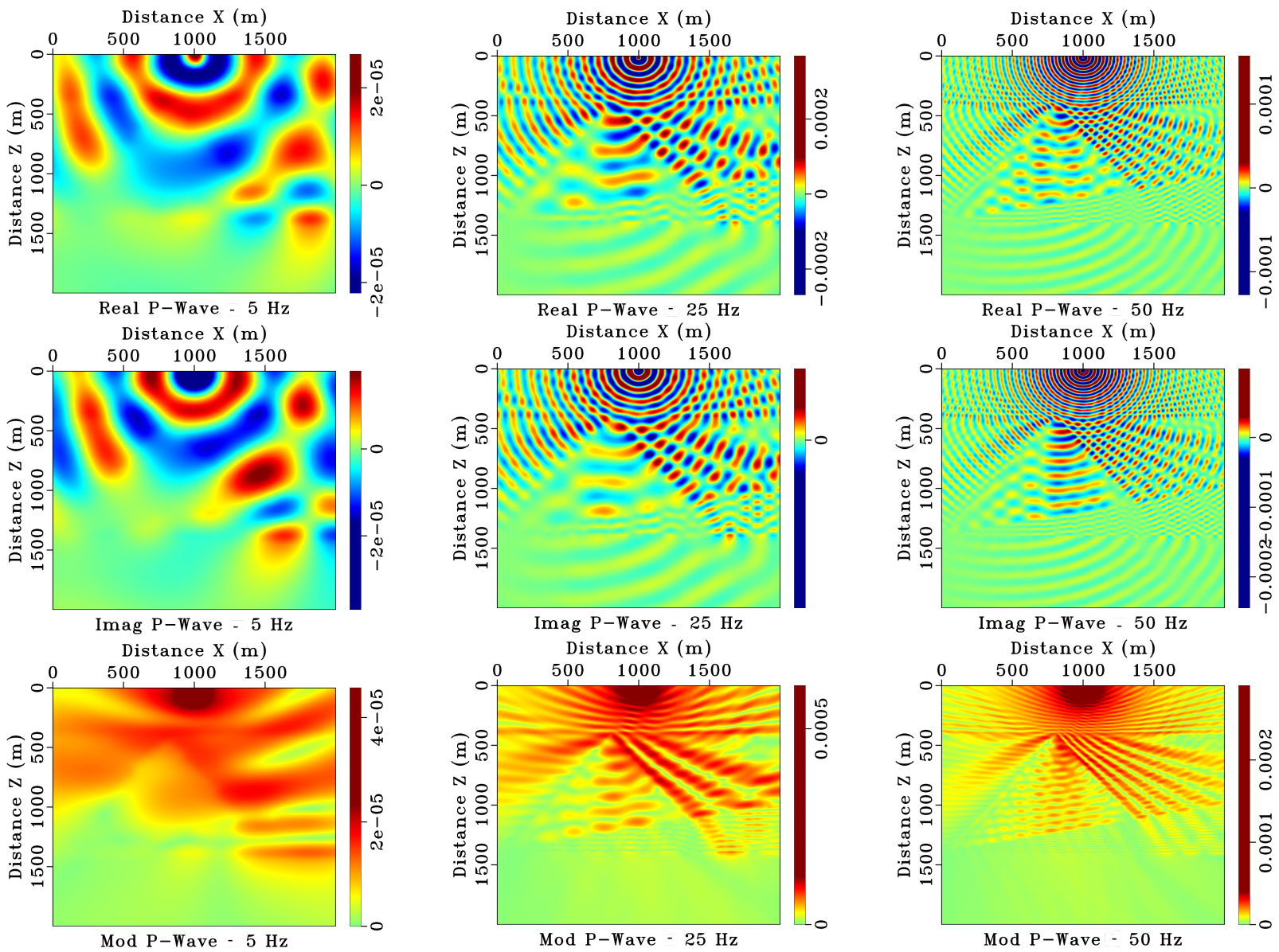

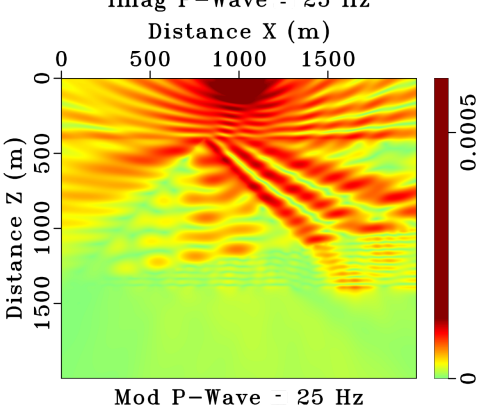

Figure 11 - Real, imaginary part and module at P-wave field for $25 \mathrm{~Hz}$.

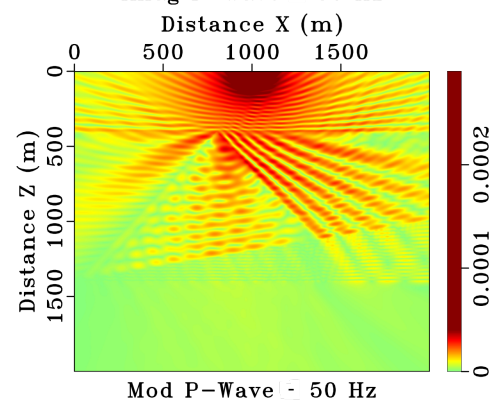

Figure 12 - Real, imaginary part and module at P-wave field for $50 \mathrm{~Hz}$.
Figure $\mathbf{1 0}$ - Real, imaginary part and module at P-wave field for $5 \mathrm{~Hz}$. 


\section{ACKNOWLEDGMENTS}

This work is supported by Colombian Oil Company (ECOPETROL) and COLCIENCIAS as a part of the research project grant No. 0266-2013. JCMC wants to thank to "Estrategia de sostenibilidad, Universidad de Antioquia". The authors want to acknowledge the anonymous referees for their observations to the document.

\section{REFERENCES}

AVENDAÑO SK. 2017. Full Waveform Inversion (FWI) in frequency Domain for the wave propagation for Visco-acoustic medium applied in synthetic data. Master dissertation. Physics. Universidad de Antioquia. Available on: <http://www.udea.edu.co/wps/wcm/connect/udea/ a429bbe8-369a-4b0c-8225-e29978fa195c/Informe_vsmall.pdf?MOD= AJPERES\&CVID=mDQYHxx>. Access on: June, 2017, 129 pp.

BERENGER JP. 1994. A perfectly matched layer for the absorption of electromagnetic waves. Journal of Computational Physics, 114: $185-200$

CARCIONE JM, HERMAN GC \& KROODE AT. 2002. Seismic modeling. Geophysics, 67: 1304-1325.

CHEN JB. 2004. Laplace-Fourier-domain dispersion analysis of an average derivative optimal scheme for scalar-wave equation. Geophys. J. Int., 197: 1681-1692.

DERAEMAEKER A BABUSKA I \& BOUILLARD P. 1999. Dispersion and pollution of the FEM solution for the Helmholtz equation in one, two and three dimensions. International Journal for Numerical Methods in Engineering, 46: 471-499.

DUTTA G, LU K, WANG X \& SCHUSTER G. 2013. Attenuation compensation in least-squares reverse time migration using the visco-acoustic wave equation. In: SEG Houston 2013 Annual Meeting. Volume 79, p. S251-S262. Houston, USA.

FICHTNER A. 2011. Full Seismic Waveform Modelling and Inversion. Berlin: Springer. 343 pp.

JO CH. 1996. An optimal 9-point, finite-difference, frequency-space, 2-D scalar wave extrapolator. Geophysical Journal, 61: 529-537.
KAMEI R \& PRATT RG. 2013. Inversion strategies for visco-acoustic waveform inversion. Geophysical Journal International, 194(2): 859-884. doi: 10.1093/gj/ggt109.

KOLSKY H. 2003. Stress Waves in Solids. Dover Phoenix Editions. Dover Publications. $212 \mathrm{pp}$.

LAVERGNE M. 1986. Seismic Methods. Editions Technip. 167 pp.

MARFURT K. 1984. Accuracy of finite-difference and finite-element modelling of the scalar and elastic wave equations. Geophysics, 49: 553-559.

MASE GT \& MASE GE. 1999. Continuum Mechanics for Engineers. Boca Raton, Florida: CRC Press. 400 pp.

MAVKO G, MUKERJI T \& DVORKIN J. 2009. The Rock Physics Handbook. Volume II. California: Cambridge University Press. 329 pp.

OPERTO S \& VIRIEUX J. 2006. Practical aspects of Frequency-domain finite-difference modelling of Seismic wave propagation. Ecole thématique SEISCOPE. 87 pp.

OSPINA MA. 2016. Dispersión y Estabilidad en el procesos demodelado en Full Wave Inversion para un medio visco-acustico en el dominio de la frecuencia en dos dimensiones. Master dissertation, Mathematics. Universidad de Antioquia, Colombia. Available on: <http: //www.udea.edu.co/wps/wcm/connect/udea/19f488d1-8b01-4b93b4b2-06b506efa4e4/Tesis.pdt?MOD=AJPERES\&CVID=mDVAVtz>. Access on: March, 2016, 87 pp.

SHIN C \& HO CHA Y. 2008. Waveform inversion in the Laplace domain. Geophys. J. Int., 173: 922-931.

STRIKWERDA J. 2007. Finite Difference Schemes and Partial Differential Equations. Philadelphia: Society for Industrial and Applied Mathematics. $434 \mathrm{pp}$.

WANG H, ZHANG L \& MA Z. 2003. Seismic wave imaging in visco-acoustic media. Science in China Series A: Mathematics, 47: 146-154.

WANG Y. 2009. Seismic Inverse Q Filtering. Wiley. 248 pp.

YANG Z, LIU Y \& REN Z. 2014. Comparisons of visco-acoustic wave equations. Journal of Geophysics and Engineering, 11: 025004. 